\title{
Collaborative Studio in a Virtual Environment
}

\author{
Marc Aurel Schnabel \\ Department of Architecture; The University of Hong Kong \\ marcaurel@hku.hk
}

\begin{abstract}
Recently, Virtual Environments (VE) are increasingly used as settings for design and research. Using VE to visualize ideas from the initial steps of design, the architect is challenged to deal with perception of space, solid and void, without translations to and from a two dimensional media. The goal of our studies was to identify how designers use and communicate early design ideas by using immersive three-dimensional (3D) VEs and how they describe $3 D$ volumes using a different media. A series of experiments were undertaken, including navigation- and perception-tasks, designing in IVE, transcription of design, remote communication between design partners and controlled observations. We explored initial intentions of 3D-immersive design schemes, textual descriptions and collaborations within IVE. We discuss frameworks and factors influencing how architectural students communicate their proposals in an immersive Virtual Environment Design Studio, and how this approach of design studio enables to understand volumes and spatial relationships.
\end{abstract}

\section{Introduction}

The design studio is a well-established context for architectural learning. Collaborative learning and designing have been demonstrated to support effective learning in architectural design teaching (Kvan et al., [10]). Architectural design is increasingly taking place in Virtual Environments (VE) (Bertol, [1]). Not only equipment and software become available and affordable for common users but also designers recognize $\mathrm{VE}$ as a tool of form-finding (Leach, [11]). However, research on the outcome of results and its possibilities of architectural design within VE is still in progress (Stuart, [15]) and lessons learned from academic contexts have already been employed in commercial settings. Architectural design teaching uses the method of Virtual Design Studios (VDS) as a common mean of instruction. While some have been successful, various issues have been reported, for example a lack of communication and collaboration (Kvan, [8]); technology overhead (Kruijff, [7]); and potential contributions to design outcomes. Immersive Virtual Environments (IVE) has not been used for design learning, although shared immersive virtual spaces have been employed for design reviews (Davidson, et al., [4]). The next step is, to establish joint design sessions where users can collaboratively create, interpret and communicate design ideas within an immersive Virtual Environment Design Studio (VeDS) and to examine if this context offers any new opportunities or solutions to problems encountered. This exercise resulted in conceptual descriptions of space. The studio is online for further information (VeDS, [16])

\section{The Virtual Environment Design Studio}

VE offers and challenges the architect to deal with perception of solid and void, navigation and function, without translations to and from a two-dimensional (2D) media (Campbell, [3]) in order to envision ideas. The goal of our studies was to identify how architects use and communicate design ideas by using IVEs.

\subsection{Experiment}

To investigate the context of a VE, we sought tasks that engaged designers at a variety of levels of complexity: the design of a commercial helicopter landing station in an urban setting. This task required users to work in three dimensions at all times yet could be abstracted to reduce representational problems. The helipad is a typical architectural task rife with complexities of functional needs (sight lines, access, form, etc.) yet also very much a three-dimensional (3D) question. This experiment including navigation- and perception challenges was conducted with remote communication between design partners and controlled observations permitting transcription of design. That allows comparison of the results with earlier experiments (Kvan, [8]).

Firstly we wanted to see if a virtual studio could be run in an immersive environment. Secondly, we wanted to see if the use of immersive virtual reality-design-systems shifted design and its communication to a different mode or level. It has been suggested, for example, that participants in a VE might express and communicate their intentions, ideas and designs not only in a different but also in an improved manner (Dorta \& LaLande, [5]). We hypothesized that the VeDS would have a positive impact 
on the development of design, its communication and understanding.

This experiment builds upon virtual studios in which the Department of Architecture at The University of Hong Kong participated since several years (Bradford, et al., [2]; Kvan, et al., [8]). Teams on the either ends worked together on the very same design task. It is a short exercise, which finishes within a single day. In short and frequent intervals the remote partner exchange design ideas, proposals and modifications, which reminded us of ping-pong match. Each side had the authority (not ownership) over parts of the design. This co-ordination is necessary in order not to obstruct the team partner's activity. A typical collaborative scenario where architects and specialists contribute to an overall scheme in sequential and parallel activities is simulated with this setup (Kvan, [8]).

Akin to brainstorming and concept finding activities, the studio focused on the initial stages of design; elaborated final designs were not the intension of the exercise. Comparable to a moderated verbal discussion in which the microphone is passed to speakers, the Head Mounted Display (HMD) was pass ed between the teams and the resultant design sketches were produced within the IVE in the course of the alternating sessions. Text communication was provided in order to support the design process. Since we wanted to capture the intent of the design, we used a modified "think aloud" methodology. We established a design team of two at each end, one wearing the HMD while the other taking notes and chatting with the remote team to convey the intent of the design in writing. These text records provided a protocol, which is analysed later on.

The VeDS used the following equipment as technical set-up on each side: two Pentium III computers with broadband internet connection, flat-screen monitors, a Kaiser Proview 60 HMD, Polhemus Fastrak magnetic tracking device and a Stylus. The Virtual Reality Architectural Modeller (VRAM) developed by Regenbrecht, et al. [12] had been modified and equipped with input features based on gesture-recognition. Similar as the input-system for $P D A$-devices, the users now gesture with the stylus and their movements are translated into basic 3D primitives (Figure 1). In order not to hinder the work done within the IVE, a separate PC was used for the communication using $I C Q$-Software, internet-browser (IE), web-based database and other presentation-software (AutoDesk 3DStudio VIZ and Adobe Photoshop).

For our experiment it was pre-requisite that the participating students acquired both a broad training in IT (through CAAD- and DTP-software) as well as an advanced background in architectura 1 design.

The intent was to engage the students in rapid design exploration, which was completed in one continuous cycle. Each design-phase was set to 30 minutes during which one team had control over the model. Each phase was finished by file-exchange and fine-tuning/adjustment of equipment. The teams placed their models and chat conversations into a database (modelled on Hirschberg, et al. [6]).

This database holds a short presentation explaining design intentions and achievements of each stage. The remote team then continued to work on that model in order to complete their segment. After four exchanges the VeDS concluded with a final presentation and live videoconference.

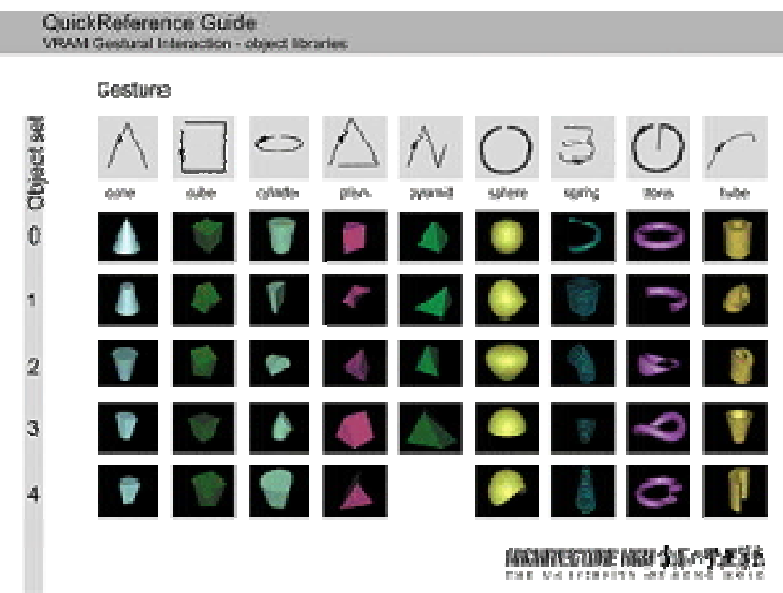

Figure 1: Gesture Reference Guide

\subsection{Results}

Most notably, we successfully demonstrated that it is possible to design, communicate and collaborate in IVEs. The eighteen participating teams did engage in collaborative work, building on the work of the team partners and own pre-ceding steps, despite the technical complexity of the system and the difficulty of working remotely together.

Next, participants noted in the chat-line communications that their designs surprised participants in their creativity and appearance. Obviously students experienced and translated their ideas differently from non-immersive environments. They stated that the interaction within IVE was direct, that each stroke had an immediate impact on the design and acts as a 'shortcut' from idea to creation. The students had the impression that they could 'communicate' directly with their model, became part of it and lost the otherwise typical distance of a designer. They reported that this led to very different forms and new arrangements in their design.

Further, collaboration was possible. We anticipated that the teamwork may not interact smoothly with each other, which occurred in earlier VDS. However, the opposite was true. The teams engaged in intense 
discussions about design, concepts and form assisted by the nature of the task and application. In order to pursue their schemes further the groups had to communicate their actions to the remote partners. However, participants developed a personal interest to share their experience and creation with their colleagues and other member of the teams.

Reviews of the graphic outcomes advocate that the students used the space actively within all three dimensions. Spatial clusters were created to represent design elements at all aspects within the available design area. Typically, an architectural design created in a 2D representation would have placed components in planview with some raised in section/elevation to achieve 3D volumes. However, in this VeDS, the students started 'sketching' their design elements at any points within the 3D sphere. Being virtually inside their design, students sculpted their proposals, making use of the flexibility of viewpoints offered in VE. They investigate the spatial impact of their design in relationship to existing forms and activities from outside and at the same time within the model (Figure 2). Though the technical constrains users rapidly learned to represent their design intent by using the cubes and spheres as representational volumes (Figure 3 ). Since these primitives can symbolize both positive and negative representations of space, other viewers of the model, were able to understand this ambiguity easily (Figure 4). In some cases, because of lack of experience or the complexity of this VE, errors or coincidences were transformed into expressive architecture (Figure 5). Other instances demonstrate that students were inspired by their own 3D model and translated their design back to a (mental) image (Figure 6). Differences in design- and operation-skills as well as architectural language can also be identified (Figure 7).

Examining the chat-conversations between the teams we anticipated a large number of navigation/orientation discussions and explanations of meaning of placed elements. Surprisingly, the analyses of the texts show only a few lines of such conversations. This suggests that participants could find their way without much difficulty and work with the IVE-interface. Students were able to understand and extract the intent behind the designscheme of the remote partner easily. The text records do not identify how or why the students were using the $3 \mathrm{D}$ space in these different ways. However, we do find evidence of intense discussion about design, functions and concepts. Students engaged in design discussion and development of the scheme by referring to the model they saw in database, submitted by their remote teamcolleagues.

The participants communicated therefore simultaneously in two ways: one being a text-based channel, through which the students discussed issues arising from their design and the other one being a model- based (virtual) channel, through which they interacted with the direct creation and arrangements of volumes and space.

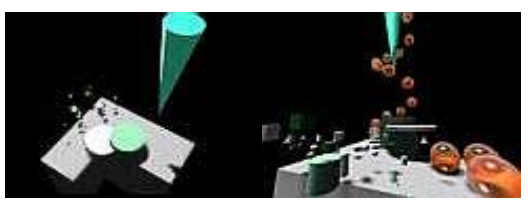

Figure 2: Users are involved (in terms of scale, viewpoint, navigation): design that uses the flexibility of VE, offers to explore structure and its spatial impacts on the creations (Playground, VeDS104)

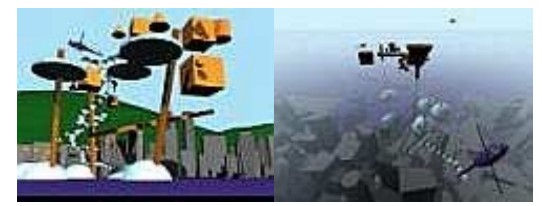

Figure 3: Primitives representing functions or forms, independently of their actual 3D shape(HeliPad, VeDS110, Phase 3 - 4)

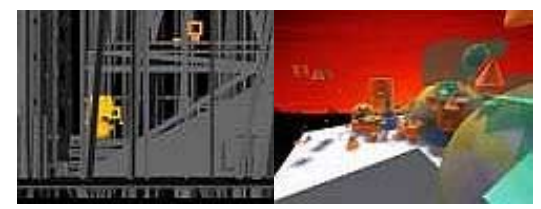

Figure 4: Primitives can symbolize both positive and negative representations of design-elements, remaining interpretable by viewers of the model (HeliPad, VeDS108, Phase 3; Playground, VeDS112)

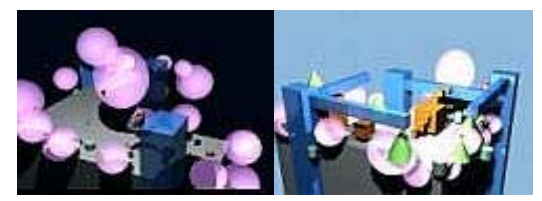

Figure 5: Lack of experiences or complexity of the VE, created errors, which are transformed into 'meaningful' architecture (Playground, VeDS103)

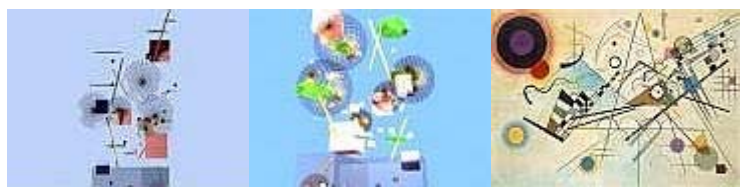

Figure 6: Plan and perspective of a design with an image by Kandinsky as mental inspiration - image added later by student (Playground, VeDS106).

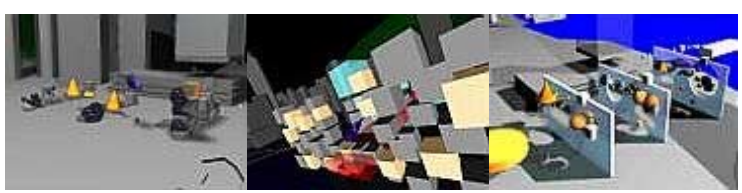

Figure 7: Differences in design- and operation-skills as well as architectural language can also be observed (HeliPad, VeD106) 
This dual channel communication enables a collaboration that is in such a way not possible using conventional design studios or non-immersive collaborative design sessions.

The complete documentation of this VeDS [16] is accessible at http://courses.arch.hku.hk/vds/veds01/db.

\section{Discussion}

According to Davidson and Campbell [4] VR is a constructive tool to support the design and communication process, at least in establishing copresence for a joint experience in spatial review. Thus far, how does this aid enlarge to a design setting? Kvan et al., [9] reported of other VDS results, which have revealed a lack of collaboration and communication. However, our experiments showed the opposite (Schnabel and Kvan, [13]). Chat-protocols show participants mentioning to each other that the team-working experience was enjoyable. Through the possibility to travel around in space, the understanding of volume and location was enhanced. Site-specific problems were not only better recognized, but also different options investigated, which is an improvement over other forms of design sharing. On one side users of IVE can change their viewpoints and escape gravity, but on the other they remain all the time 'inside' their model without having to translate immediately scales or dimensionalities. Designers can therefore work more three-dimensionally since every object within the VE is experienced through movement and interaction. This possibility offers a different 'conversation' with their design that otherwise is not obvious or possible. Spatial issues are addressed in a manner akin to the real world. The tools enhancing the translation of the designers' and users' mental intention, experiences that were encountered perhaps in spite of the technology used and the abstractness of VE.

This research builds upon prior experiments in communication between designers in $\mathrm{VE}$ and how they collaborate with partners to solve 3D tasks. We first reduced the question of volumetric understanding to an abstract problem solving task in order to test issues that may rise for the VeDS, then we carried out an architectural virtual design studio that took issues of VE to a more realistic architectural design scenario (Schnabel et al, [14]). Our findings support our assumption. We find that it is important for architects to use in the early design stages a tool that reflects the three-dimensionality of their design such as VE. Using a 2D medium to translate spatial ideas apparently reduces the exploration and communication of volume and space. We demonstrated this with our design example of the heliport and the abstract description of the 3D cube. Designing within and understanding a 3D space, IVE offers new opportunities of languages to designers. Consequently, the field is too rich to cover all aspects in these researches.

IVE offers a new form of teamwork. Architects can collaborate with colleagues or clients using an interactive media, which supports the design and communication process in a more immediate way. Communication is enhanced through a media, which relates to the process of thinking, creation and understanding. Traditionally architectural design is a single creator and single user domain. Even within a teamwork, explicit tasks are specified and solved individually. Our VeDS established a unique combination of collaboration and communication of an interactive design process, which is more transparent and imminent. Involved parties worked together in order to come up with the desired solution. Most importantly, the VeDS shows that users of an IVE do indeed 'read' volumes and spatial relationships better than when working in $2 \mathrm{D}$ representations.

Our experiment has shown that IVE can support an instantaneous, direct, scale-less and intuitive control over a 3D design. However, as of today, capabilities of VE software do not match the sophistication of today's CAD software as well as the predominant training of architects to translate and read 2D plans, representing 3D space. Therefore VE can supplement, but not replace, other design media. An immersive and easy-manageable environment is needed before immersive VR can change effectively the design process outside our research conditions. This can then be used broadly in normal architectural and related applications.

However, it is not as simple as just placing a designer in a VE. Postulations about what works and what does not need to be tested. Technology issues such as usability interface and navigation and have to be further developed to reach the same ease to use and familiarity as any $2 \mathrm{D}$ media. Problems with the working environment clearly limited what the designers could do. In particular, clumsiness of gesturing and limited field of vision constrained use. Particular problems encountered were the wiring of HMD and tracker entangling arms or legs; interference of and sensitivity of the tracker; lack of precision in gesture recognition and insert-points of elements; polygon size of models; frame rate of display, rendering and calculation time of models; cost of equipment; inability to support multi-user, multiviewpoints and networking of VEs are all issues that deserve attention.

\section{Conclusion}

A 'virtual design studio' was successfully conducted. Pairs of students formed teams and two teams worked across the network to develop sequentially a design in an immersive environment. In this study, the procedure was observed to identify the achieved spatial-understanding 
and the degree of communication. The experiment has confirmed that design within IVEs can lead to meaningful and new architectural results. The direct feedback of cause and effect of VE in the design process and the enhanced teamwork offers architects a new way to explore, design, interact and communicate spatial constructions. The understanding and description of complex volumes is improved within an IVE setting.

Since IVEs play increasingly a role in the design and form finding of architectural creation, virtuality becomes, in that sense, reality. Working in VE architects can explore alternative solutions to those achieved in conventional design methods, despite those issues of visual perception, mental images/workload, errors, comprehension of design and its communication, frequency of creation/feedback/modification-loops as well as impact on the design-creation. Our experiments demonstrate that, the problems of VE are not terminal, preventing effective collaboration, nor are they permanent. Because technical solutions are constantly evolving, difficulties resolved and equipment is becoming more sophisticated, affordable and easy to use, IVE give designers a set of tools (such as other technologies, for example rapid prototyping and automated construction methods), with which they can articulate different ideas in a for most users simple manner. VE permits users to create, visualize and communicate ideas with the help of an effective tool. This successful experiment also opens new possibilities for experts and novices to collaborate and communicate their ideas and wishes.

\section{Acknowledgements}

We sincerely appreciate the time and energy everybody expended in support and sustain of this first VeDS. Recognition is given to the students who took part in the design studio and willingly gave of their thoughts and designs. Financial backing came from the Germany/Hong Kong Joint Research Scheme.

\section{References}

[1] Bertol, D.: 1997, Designing digital space: an architect's guide to virtual reality New York, John Wiley \& Sons Ltd.

[2] Bradford, J. W., Cheng, N. \& Kvan, T.: 1994, Virtual Design Studios, in T. Maver \& J. Petric (eds), The Virtual Studio, eCAADe Conference Proceedings, University of Strathclyde, Glasgow, UK, 7-10 September 1994, pp. 163-167

[3] Campbell, D. A.: 1996, The Nature of Cyberspace; Vers Une Architecture Virtuelle...; Crit 35;

http://www.hitl.washington.edu/people/dace/portfoli/crit35.html (Accessed 12/08/2002).
[4] Davidson, J. N. and Campbell, D. A.: 1996, Collaborative Design in Virtual Space - GreenSpace II: A Shared Environment for Architectural Design Review, in Design Computation: Collaboration, Reasoning, Pedagogy, ACADIA Conference Proceedings, Tucson, USA, 31/10-2/11/96, pp. 165179

[5] Dorta, T. and LaLande, P.: 1998, The impact of virtual reality on the design process, in S. van Wyk and T. Seebohm (eds), Digital Design Studios: Do Computers Make a Difference in Design Studio? ACADIA Conference Proceedings 1998, Québec City, Québec, Canada, 22-25 October, pp. 138-160

[6] Hirschberg, U., Schmitt, G., Kurmann, D., Kolarevic, B., Johnson, B. and Donath, D.: 1999, The 24 Hour Design Cycle: An Experiment in Design Collaboration over the Internet, in Proceedings of The Fourth Conference on Computer Aided Architectural Design Research in Asia, CAADRIA '99, Shanghai, China, 5-7 May 1999, pp. 181-190

[7] Kruijff, E.: 1998, VDS '97 Project Report on the Multiplying Time VDS '97 project, Utrecht University, The Netherlands, http://kwetal.ms.mff.cuni.cz/ ernst/intro.htm (Accessed May 2001)

[8] Kvan, T.: 2000, Collaborative design: what is it? Automation in Construction 9(4) (2000) 409-415. New York, Elsevier

[9] Kvan, T., Schmitt, G. N., Maher, M. L. and Cheng, N.: 2000, Teaching Architectural Design in Virtual Studios, in Computing in Civil and Building Engineering, R. Fruchter, F. Peña-Mora \& W. M. K. Roddis (eds). Eight International Conference on Computing in Civil and Building Engineering (ICCCBE-VIII), vol. 1, vol. 1, Stanford: ASCE, pp. 162-169

[10] Kvan, T., Yip A.and Vera, A.,: Supporting Design Studio Learning: An investigation into design communication in computer-supported collaboration, CSCL'99, Stanford, December 1999, pp 328-332

[11] Leach, Neil: 2002, Designing for a Digital World (Architectural Design), New York, John Wiley \& So.

[12] Regenbrecht, H., Kruijff, E., Donath, D., Seichter, H. and Beetz, J.: 2000, VRAM - A Virtual Reality Aided Modeller, Promise and Reality, eCAADe Conference Proceedings, Weimar, Germany, 22-24 June 2000, pp. 235-237

[13] Schnabel, M. A. \& Kvan, T.: 2001, Implementing The First Virtual Environment Design Studio, Architectural Education for the Asian Century, Proceedings of the 1st ACAE Conference on Architectural Education, Milton Tan, editor, Centre for Advanced Studies in Architecture, National University of Singapore, 2001, pp 157-166

[14] Schnabel, M. A., Kvan, T., Kruijff, E. and Donath, D.: 2001, The First Virtual Environment Design Studio; Architectural Information Management, 19th eCAADe Conference Proceedings, Hannu Penttilä, ed., Helsinki (Finland) 29-31 August 2001, pp. 394-400.

[15] Stuart, Rory: 1996, The design of virtual environments. New York, McGraw-Hill

[16] VeDS: 2001, Virtual Environment Design Studio at the Department of Architecture, The University of Hong Kong, http://courses.arch.hku.hk/vds/veds01/db (Accessed 12 /08/2002) 